\title{
Menger's Theorem for Matroids
}

\author{
W. T. Tutte \\ Professor of Mathematics, University of Waterloo, Ontario, Canada
}

(November 16, 1964)

\begin{abstract}
Menger's Theorem asserts that if $x$ and $y$ are vertices of a graph which are not joined by an edge and if it takes at least $k$ other vertices to separate $x$ and $y$, then $x$ and $y$ can be joined by $k$ distinct arcs in the graph which have only their end-vertices in common.

Much of the paper is taken up by the determination of an equivalent statement involving the ranks of subgraphs of contractions of the original graph, but not explicitly mentioning vertices. An analogous proposition for matroids can then be stated. For matroid theory has analogs of subgraphs and contractions, but not of vertices. Once stated the generalized theorem can be proved without great difficulty.
\end{abstract}

\section{Statement of the Theorem}

In this paper we state and prove a theorem about general matroids which can be regarded as a generalization of Menger's Theorem for graphs. We state this theorem in the present section. The remainder of the paper falls into two parts. The first part, constituting section 2 , is concerned with justifying the claim that Menger's Theorem can be deduced from our matroid theorem as a special case. The second part is concerned with the proof of the theorem. Thus a reader interested in general matroids but not in graphs might omit section 2 .

Let $M$ be a matroid on a set $E$, and let $X$ and $Y$ be disjoint subsets of $E$.

If $\{S, T\}$ is any partition of $E$ we write

$$
\begin{aligned}
& \xi(M, S, T) \\
= & r(M)-r(M \times S)-r(M \times T)+1 .
\end{aligned}
$$

We also write $k(M ; X, Y)$ for the minimum of $\xi(M ; S, T)$, taken over all partitions $\{S, T\}$ of $E$ for which $X \subseteq S$ and $Y \subseteq T$.

We define $h(M ; X, Y)$ as the maximum of $\xi\left(M^{\prime} ; X, Y\right)$, taken over all minors $M^{\prime}$ of $M$ of the form $(M \times S) \cdot(X \cup Y)$.

The theorem to be proved is

1.1

$$
h(M ; X, Y)=k(M ; X, Y) \text {. }
$$

\section{Menger's Theorem for Graphs}

Let $x$ and $y$ be distinct vertices of a graph $G$ which are not joined by an edge. A set $S \subseteq V(G)-\{x, y\}$ is said to separate $x$ and $y$ if each path from $x$ to $y$ in
$G$ passes through a vertex of $S$. The least possible number $|S|$ of members of such a set $S$ will be denoted by $k(G ; x, y)$.

A path in $G$ is called simple if it passes through no edge or vertex more than once.

Let $\left\{P_{1}, P_{2}, \ldots, P_{h}\right\}$ be a set of $h$ simple paths in $G$. They are disjoint if no two have a common edge or vertex. If they are all paths from $x$ to $y$ we say they are internally disjoint if no two of them have a common edge or vertex apart from the two vertices $x$ and $y$.

We write $h(G ; x, y)$ for the maximum number of internally disjoint simple paths in $G$ from $x$ to $y$. Menger's Theorem, in one version, asserts that

$$
h(G ; x, y)=k(G ; x, y) .
$$

The theorem can be stated in several equivalent though apparently different forms. The most convenient for our purposes is the following.

We replace $x$ and $y$ by disjoint non-null sets $X$ and $Y$ of edges of $G$. We define $k(G ; X, Y)$ as the smallest number of vertices forming a set $S$ which separates $X$ and $Y$, that is such that each simple path in $G$ with one end a vertex of $G \cdot X$ and the other of $G \cdot Y$ passes through a vertex of $S$. (Here we use the notation of sec. 3.1 of "Lectures on Matroids".)

We now define $h(G ; X, Y)$ as the maximum number of disjoint simple paths joining $X$ and $Y$, that is having one end in $V(G \cdot X)$ and the other in $V(G \cdot Y)$. It must be borne in mind that a "simple" path may be degenerate, that is consist of a single vertex.

Our second version of Menger's Theorem asserts that

$$
h(G ; X, Y)=k(G ; X, Y) \text {. }
$$


To deduce 2.1 from 2.2 we take $X$ and $Y$ to be the sets of edges incident with $x$ and $y$ respectively. The derivation of 2.2 from 2.1 becomes obvious after the following construction is made. We introduce new vertices $x$ and $y$, and join them to all the vertices of $G \cdot X$ and $G \cdot Y$ respectively by new edges.

It might seem more natural to take $X$ and $Y$ as sets of vertices rather than edges. This is done, for example, by König. But then no generalization to matroids is suggested, since matroids have no vertices.

In discussions of Theorem 2.2 there is no real loss of generality in supposing $G \cdot X$ to be connected. For we can make it so by adjoining new edges, each to both $G$ and $X$, and this operation does not affect the numbers $h(G ; X, Y)$ and $k(G ; X, Y)$. There is also no harm in assuming $G \cdot Y$ connected.

We may also assume $G$ connected. For in all nontrivial cases the connected subgraphs $G \cdot X$ and $G \cdot Y$ are contained in the same component $H$ of $G$, and we may replace $G$ by $H$.

Let us try to give a definition of $k(G ; X, Y)$ which does not mention vertices.

If $S$ and $T$ are complementary subsets of $E(G)$ we define $\eta(G ; S, T)$ as the number of common vertices of $G \cdot S$ and $G \cdot T$. We next define $\mu(G ; X, Y)$ as the minimum value of $\eta(G ; S, T)$, when $S$ and $T$ are restricted only by the conditions $X \subseteq S$ and $Y \subseteq T$.

$$
\mu(G ; X, Y)=k(G ; X, Y) .
$$

Proof: If $S$ and $T$ are defined as above, then each simple path joining $X$ and $Y$ has its first vertex incident with a member of $S$ and its last with a member of $T$. Hence one of its vertices is incident with members of both $S$ and $T$. We deduce that the common vertices of $G \cdot S$ and $G \cdot T$ form a set separating $X$ and $Y$. Thus $\mu(G ; X, Y) \geqslant k(G ; X, Y)$.

Conversely suppose we have a set $Q$ of vertices separating $X$ and $Y$. Let $S$ be the set of all edges $A$ of $G$ such that some simple path $P$ traversing $A$ has the following properties.

(i) The first vertex of $P$ is incident with an edge of $X$.

(ii) No vertex of $P$, save possibly the last, belongs to $Q$.

It is clear that $S \cap Y$ is null and that if $T=E(G)-S$ then any common vertex of $G \cdot S$ and $G \cdot T$ is in $Q$. Hence $\mu(G ; X, Y) \leqslant k(G ; X, Y)$, since as a special case we may have $|Q|=k(G ; X, Y)$.

The theorem follows.

2.4 Suppose $G, G \cdot X$ and $G \cdot Y$ are connected. Let $\{S, T\}$ be a partition of $E(G)$ such that $X \subseteq S$ and $Y \subseteq T$. Then

$$
\begin{aligned}
& \eta(G ; S, T)-k(G ; X, Y) \\
\geqslant & p_{0}(G \cdot S)+p_{0}(G \cdot T)-2 .
\end{aligned}
$$

Proof: Assume that the theorem fails, and that $p_{0}(G \cdot S)+p_{0}(G \cdot T)$ has the least value consistent with this condition.
Clearly $p_{0}(G \cdot S)+p_{0}(G \cdot T) \geqslant 3$, by 2.3 .

Without loss of generality we may suppose $G \cdot S$ to have a component $G \cdot S_{1}$ which does not meet $G \cdot X$. Let this component meet $q$ components of $G \cdot T$ and let it have $p$ common vertices with $G \cdot T$. Then, abbreviating $p_{0}(G \cdot U)$ as $p_{0}(U)$, we have the following relationships.

$$
\begin{array}{ll}
\text { (i) } & 0<q \leqslant p . \\
\text { (ii) } & p_{0}\left(S-S_{1}\right)=p_{0}(S)-1 \\
\text { (iii) } & p_{0}\left(T \cup S_{1}\right)=p_{0}(T)-q+1, \\
\text { (iv) } & \eta\left(G ; S-S_{1}, T \cup S_{1}\right) \\
& =\eta(G ; S, T)-p .
\end{array}
$$

The inequality $0<q$ follows from the fact that $G$ is connected.

We now have

$$
\begin{aligned}
& \eta(G ; S, T)-k(G ; X, Y) \\
= & \eta\left(G ; S-S_{1}, T \cup S_{1}\right)-k(G ; X, Y)+p, \\
\geqslant & p_{0}\left(S-S_{1}\right)+p_{0}\left(T \cup S_{1}\right)+p-2,
\end{aligned}
$$

by the choice of $S$ and $T$. For

$$
p_{0}\left(S-S_{1}\right)+p_{0}\left(T \cup S_{1}\right)<p_{0}(S)+p_{0}(T),
$$

by (i), (ii), and (iii). Hence

$$
\begin{aligned}
& \eta(G ; S, T)-k(G ; X, Y) \\
& \geqslant p_{0}(S)+p_{0}(T)+p-q-2,
\end{aligned}
$$

by (ii) and (iii),

$$
\geqslant p_{0}(S)+p_{0}(T)-2
$$

But this is contrary to assumption. The theorem follows.

If $G$ is a graph of $p_{0}(G)$ components the rank of its bond-matroid $B(G)$ can be deduced from Theorem 2.21 of "Matroids." A principal forest of $G$ consists of $p_{0}(G)$ disjoint trees, and in any tree the number of vertices exceeds the number of edges by 1 . So by the theorem just quoted we have

$$
r(B(G))=|V(G)|-p_{0}(G)
$$

Now let $S$ and $T$ be complementary subsets of $E(G)$. Using Theorem 3.321 of "Matroids" we find that

$$
\begin{aligned}
& r(B(G))-r(B(G) \times S)-r(B(G) \times T) \\
= & r(B(G) \cdot T)+r(B(G) \cdot S)-r(B(G)),
\end{aligned}
$$


by "Matroids," 3.54,

$$
\begin{aligned}
& =V(G \cdot T)+V(G \cdot S)-V(G) \\
& =p_{0}(G \cdot T)-p_{0}(G \cdot S)+p_{0}(G),
\end{aligned}
$$

by "Matroids," 3.321,

$$
\begin{aligned}
& =\eta(G ; S, T)+p_{0}(G) \\
& -p_{0}(G \cdot S)-p_{0}(G \cdot T),
\end{aligned}
$$

if $G$ has no isolated vertices.

2.7 If $G, G \cdot X$ and $G \cdot Y$ are connected, then $k(G$; $X, Y)$ is the minimum of

$$
r(B(G))-r(B(G) \times S)-r(B(G) \times T)+1,
$$

taken over all partitions $\{S, T\}$ of $E(G)$ such that $X \subseteq S$ and $Y \subseteq T$.

Proof: Denote this minimum by $m$. Applying 2.4 to the result just proved we find that $m \geqslant k(G ; X, Y)$. Moreover if $\eta(G ; S, T)=k(G ; X, Y)$ we have $p_{0}(G \cdot S)$ $=p_{0}(G \cdot T)=1$, by 2.4 , and therefore

$$
\begin{aligned}
& r(B(G))-r(B(G) \times S)-r(B(G) \times T)+1 \\
= & \eta(G ; S, T)=k(G ; X, Y) .
\end{aligned}
$$

The theorem follows.

Theorem 2.7 has the following corollary

$$
k(G ; X, Y)=k(B(G) ; X, Y) .
$$

It is clear that the definition of $h(G ; X, Y)$ given above is equivalent to the following; $h(G ; X, Y)$ is the maximum number of disjoint connected subgraphs of $G$, no one including an edge of $X \cup Y$, such that each has at least one vertex in common with each of the graphs $G \cdot X$ and $G \cdot Y$.

We express this result in terms of the notation of section 3.1 of "Matroids" as follows. The vertices of the graph $H=(G \times S) \cdot(X \cup Y)$, where $X \cup Y \subseteq S \subseteq E$, are the components of $G:(E(G)-S)$. They are thus disjoint connected subgraphs of $G$. The edges of $H$ are the members of $X \cup Y$. The ends of each such edge in $H$ are those vertices of that graph which, as subgraphs of $G$, contain the ends of the edge in $G$.

It follows that $h(G ; X, Y)$ is the maximum number of common vertices of $\boldsymbol{H} \cdot X$ and $H \cdot Y$, where $H=(G \times S)$ - $(X \cup Y)$ ranges over all possible choices of $S$. It is the maximum of $\eta(H ; X, Y)$, taken over all possible choices of $S$. If we assume that $G, G \cdot X$ and $G \cdot Y$ are connected we can readily verify that $H, H \cdot X$ and $H \cdot Y$ are also connected. We then have $\eta(H ; X, Y)=\xi(B(H)$; $X, Y)$, by 2.6. But $B(H)=(B(G) \times S) \cdot(X \cup Y)$. It follows that

$$
h(B(G) ; X, Y)=h(G ; X, Y) .
$$

Formulae 2.71 and 2.8 hold when $G, G \cdot X$ and $G \cdot Y$ are connected. They justify the assertion that 1.1 then includes 2.2 as a special case. The extension to the general case for graphs may be made by devices already described.

\section{Some Auxiliary Theorems}

We prove Menger's Theorem for matroids in the next section. Here we give a preliminary discussion of the functions

$\xi(M ; S, T)$ and $k(M ; X, Y)$.

3.1 Let $\{S, T\}$ be any partition of $E$. Then

$$
\xi\left(M^{*} ; S, T\right)=\xi(M ; S, T) .
$$

Proof:

$$
\begin{aligned}
& \xi(M ; S, T) \\
= & r(M)-r(M \times S)-r(M \times T)+1 \\
= & -r(M)+r(M \cdot T)+r(M \cdot S)+1,
\end{aligned}
$$

by "Matroids," 3.54,

$$
\begin{aligned}
= & -|E|+r\left(M^{*}\right)+|T|-r\left((M \cdot T)^{*}\right) \\
& +|S|-r\left((M \cdot S)^{*}\right)+1,
\end{aligned}
$$

by "Matroids," 3.52,

$$
=r\left(M^{*}\right)-r\left(M^{*} \times T\right)-r\left(M^{*} \times S\right)+1,
$$

by "Matroids," 3.351,

$$
=\xi\left(M^{*}, S, T\right) .
$$

As a Corollary we have

3.11 Let $X$ and $Y$ be disjoint subsets of $E$. Then

$$
k\left(M^{*} ; X, Y\right)=k(M ; X, Y) .
$$

3.2 Let $\{S, T\}$ and $\{U, V\}$ be partitions of $E$. Then

$$
\begin{aligned}
& \xi(M ; S \cup U, T \cap V)+\xi(M ; S \cap U, T \cup V) \\
& \quad \leqslant \xi(M ; S, T)+\xi(M ; U, V) .
\end{aligned}
$$

Proof:

$$
\begin{aligned}
& \xi(M: S \cup U, T \cap V)+\xi(M ; S \cap U, T \cup V) \\
= & 2 r(M)+2-r(M \times(S \cup U))-r(M \times(S \cap U)) \\
- & r(M \times(T \cup V))-r(M \times(T \cap V)) \\
\leqslant & 2 r(M)+2-r(M \times S)-r(M \times U) \\
- & r(M \times T)-r(M \times V),
\end{aligned}
$$


by "Matroids," 3.56

$$
=\xi(M ; S, T)+\xi(M ; U, V) .
$$

Suppose $a \epsilon S \subseteq E$. We define an integer $\theta(M ; S, a)$ as follows. $\theta(\bar{M} ; S, a)=1$ if there is an atom $X$ of $M$ such that $a \in X \subseteq S$, and $\theta(M ; S, a)=0$ otherwise. Thus

$$
\theta(M ; S, a)=r((M \times S) \cdot\{a\}) .
$$

We take note of the following trivial result.

3.3 If $a \epsilon S \subseteq T \subseteq E$, and $\theta(M ; S, a)=1$, then $\theta(M ; T, a)$ $=1$.

From now on we abbreviate $r(M \times S)$ as $r(S)$. Thus, using 3.54 of "Matroids" we have

3.4 If $\mathrm{a} \epsilon \mathrm{S} \subseteq \mathrm{E}$, then

$$
r(S)=r(S-\{a\})+\theta(M ; S, a)
$$

Given a cell $a \epsilon E$ we abbreviate the expressions $M \times(E-\{a\})$ and $M \cdot(E-\{a\})$ as $M_{a}^{\prime}$ and $M_{a}^{\prime \prime}$ respectively.

3.51 If $\{S, T\}$ is a partition of $\mathrm{E}-\{\mathrm{a}\}$ then

$$
\begin{aligned}
& \xi\left(M_{a}^{\prime} ; S, T\right) \\
= & \xi(M ; S \cup\{a\}, T)-\theta(M ; E, a)+\theta(M, S \cup\{a\}, a),
\end{aligned}
$$

by 3.4 and the definition of the function $\xi(M ; U, V)$. 3.52 If $\{S, T\}$ and a are as in 3.51, then

$$
\begin{aligned}
& \xi\left(M_{a}^{\prime \prime} ; S, T\right) \\
= & \xi(M ; S \cup\{a\}, T)+r\{a\})-\theta(M ; T \cup\{a\}, a) .
\end{aligned}
$$

Proof:

If $U \subseteq E-\{a\}$ we have

$$
r\left(M_{a}^{\prime \prime} \times U\right)=r((M \times(U \cup\{a\})) \cdot U),
$$

by "Matroids," 3.333,

$$
=r(U \cup\{a\})-r(\{a\}),
$$

by "Matroids," 3.54 .

We deduce that

$$
\begin{aligned}
& \xi\left(M_{a}^{\prime \prime} ; S, T\right) \\
= & r\left(M_{a}^{\prime \prime}\right)-r\left(M_{a}^{\prime \prime} \times S\right)-r\left(M_{a}^{\prime \prime} \times T\right)+1 \\
= & r(E)-r(\{a\})-r(S \cup\{a\})+r(\{a\}) \\
- & r(T \cup\{a\})+r(\{a\})+1 \\
= & \xi(M ; S \cup\{a\}, T)+r(\{a\}) \\
- & \theta(M ; T \cup\{a\}, a) .
\end{aligned}
$$

By 3.3 the difference $\theta(M ; E, a)-\theta(M ; S \cup\{a\}, a)$ of 3.51 is either 1 or 0 . Similarly, since $r(\{a\})=\theta(M$; $\{a\}, a)$, the difference $\theta(M ; T \cup\{a\}, a)-r(\{a\})$ of 3.52 is either 1 or 0 . Hence, by 3.51, 3.52, and the definition of $k(M ; X, Y)$ we have the following theorem.

3.6 If $X$ and $Y$ are disjoint subsets of $E$, and $a \in E-$ $(X \cup Y)$, then the following propositions hold.

(i) $k(M ; X, Y) \geqslant k\left(M_{a}^{\prime} ; X, Y\right) \geqslant k(M ; X, Y)-1$,

(ii) $k(M ; X, Y) \geqslant k\left(M_{a}^{\prime} ; X, Y\right) \geqslant k(M ; X, Y)-1$.

We can improve upon this result as follows.

3.7 If $X, Y$ and a are as in 3.6, then either

$$
k\left(M_{a}^{\prime} ; X, Y\right)=k(M ; X, Y)
$$

or

$$
k\left(M_{a}^{\prime \prime} ; X, Y\right)=k(M ; X, Y) .
$$

Proof: Suppose the theorem to fail. Then there exist partitions $\{S, T\}$ and $\{U, V\}$ of $E-\{a\}$, with $X \subseteq S \cap U$ and $Y \subseteq T \cap V$, which have the following properties.

(i) $\xi(M ; S \cup\{a\}, T)=\xi(M ; S, T \cup\{a\})$

$$
=\xi\left(M_{a}^{\prime} ; S, T\right)+1=k(M ; X, Y),
$$

(ii) $\xi(M ; U \cup\{a\}, V)=\xi(M ; U, V \cup\{a\})$

$$
=\xi\left(M_{a}^{\prime \prime} ; U, V\right)+1=k(M ; X, Y) \text {. }
$$

Using 3.51 we find

$$
\xi\left(M_{a}^{\prime} ; U, V\right) \leqslant k(M ; X, Y) .
$$

So, by (i) and 3.2,

$$
\begin{aligned}
\xi\left(M_{a}^{\prime} ;\right. & S \cup U, T \cap V)+\xi\left(M_{a}^{\prime} ; S \cap U, T \cup V\right) \\
& \leqslant 2 k(\boldsymbol{M} ; X, Y)-1 .
\end{aligned}
$$

We may therefore suppose, without loss of generality, that

(iii) $\xi(M ; S \cup U \cup\{a\}, T \cap V)$ $=\xi(M ; S \cup U,(T \cap V) \cup\{a\})$

$$
=\xi\left(M_{a}^{\prime} ; S \cup U, T \cap V\right)+1=k(M ; X, Y) .
$$

By 3.52 and (ii) we have

$$
\theta(M ; U \cup\{a\}, a)=1,
$$

and by 3.51 and (iii) we have

$$
\theta(M ; S \cup U \cup\{a\}, a)=0 .
$$

But these results contradict 3.3. The theorem follows.

3.8 If $X, Y$, and a are as in 3.6, then

$$
h(M ; X, Y)=\operatorname{Max}\left[h\left(M_{a}^{\prime} ; X, Y\right), h\left(M_{a}^{\prime} ; X, Y\right)\right] .
$$


Proof. Consider a minor $M_{1}=(M \times S) \cdot(X \cup Y)$ of $M$. If $a \notin S$ we can write $M_{1}=\left(M_{a}^{\prime} \times S\right) \cdot(X \cup Y)$. If however $a \epsilon S$ we have

$$
\begin{aligned}
M_{1} & =((M \times S) \cdot(S-\{a\})) \cdot(X \cup Y) \\
& =\left(M_{a}^{\prime \prime} \times(S-\{a\})\right) \cdot(X \cup Y) .
\end{aligned}
$$

Conversely a minor $M_{2}$ of $M_{a}^{\prime}$ of the form $\left(M_{a}^{\prime} \times S\right)$. $(X \cup Y)$ can be rewritten as $(M \times S) \cdot(X \cup Y)$. For a minor $M_{2}=\left(M_{a}^{\prime \prime} \times S\right) \cdot(X \cup Y)$ we have

$$
\begin{aligned}
M_{2} & =((M \cdot(E-\{a\})) \times S) \cdot(X \cup Y) \\
& =(M \times(S \cup\{a\})) \cdot(X \cup Y) .
\end{aligned}
$$

Hence the minors of $M$ of the form $(M \times S) \cdot(X \cup Y)$ are the minors of $M_{a}^{\prime}$ of the form $\left(M_{a}^{\prime} \times S\right) \cdot(X \cup Y)$ together with those of $M_{a}^{\prime \prime}$ of the form $\left(M_{a}^{\prime \prime} \times S\right) \cdot(X \cup Y)$. The theorem follows.

\section{Proof of the Main Theorem}

We proceed to prove the proposition already stated as 2.1 , namely that

$$
h(M ; X, Y)=k(M ; X, Y)
$$

for arbitrary disjoint subsets $X$ and $Y$ of $E$.

Write $m=|E-(X \cup Y)|$. If $m=0$ the theorem is trivially true, since both $h(M ; X, Y)$ and $k(M ; X, Y)$ are equal to $\xi(M ; X, Y)$.

Assume as an inductive hypothesis that it is true whenever $m$ is less than some positive integer $q$ and consider the case $m=q$.

Choose $a \epsilon E-(X \cup Y)$. Then

$$
k(M ; X, Y)=\operatorname{Max}\left[k\left(M_{a}^{\prime} ; X, Y\right), k\left(M_{a}^{\prime \prime} ; X, Y\right)\right],
$$

by 3.6 and 3.7 ,

$$
=\operatorname{Max}\left[h\left(M_{a}^{\prime} ; X, Y\right), h\left(M_{a}^{\prime \prime} ; X, Y\right)\right],
$$

by the inductive hypothesis,

$$
=h(M ; X, Y),
$$

by 3.8 .

Thus the theorem holds also when $m=q$. It fol(Paper 69B1-132) lows in general by induction. 